e-migrinter

\author{
e-Migrinter
}

16 | 2017

Récits d'exilés

\title{
Récits de vie de femmes migrantes : vers des écritures plurielles de soi
}

\section{Delphine Leroy}

\section{(2) OpenEdition}

\section{Journals}

Édition électronique

URL : https://journals.openedition.org/e-migrinter/945

DOI : 10.4000/e-migrinter.945

ISSN : 1961-9685

Éditeur

UMR 7301 - Migrinter

Référence électronique

Delphine Leroy, « Récits de vie de femmes migrantes : vers des écritures plurielles de soi », e-Migrinter [En ligne], 16 | 2017, mis en ligne le, consulté le 20 mai 2021. URL : http://journals.openedition.org/emigrinter/945; DOI : https://doi.org/10.4000/e-migrinter.945

Ce document a été généré automatiquement le 20 mai 2021.

Tous droits réservés 


\title{
Récits de vie de femmes migrantes : vers des écritures plurielles de soi
}

\author{
Delphine Leroy
}

1 Produire un récit de soi à l'âge adulte, dans une langue qui n'est pas celle utilisée lors de l'enfance, telle est l'expérience relatée par des femmes migrantes en France au début de ce second millénaire.

2 A travers des écritures ordinaires, des récits biographiques publiés ou non et des récits oraux, ces scriptrices ne cessent d'évoquer le rapport à l'écriture comme un rapport biographique aux savoirs et à l'apprentissage. Cette "biographisation » (DeloryMomberger, 2014) de la trace et du récit de soi s'inscrivent dans l'expérience migratoire. Des évocations de ce parcours apparaissent de manière visibles ou audibles (par l'usage simultané de différentes langues, d'un accent) ou bien de manière plus intériorisées (par exemple la musicalité de la prose en français d'auteur-e-s venus d'ailleurs ou leurs usages marginaux de certains termes évoquent une forme de « créolité » de la langue à la manière d'Edouard Glissant). Une référence explicite à ce mouvement, ce changement géographique, linguistique et les bouleversements personnels qu'il a occasionnés préside de manière constante à leurs narrations.

Dès lors, analyser la situation d'écriture et d'«auteurisation » pour des femmes migrantes en France est une manière de se centrer sur la personne qui fait ressurgir ses particularités, dans lesquelles l'expérience migratoire et la singularité de son expression sont particulièrement enchâssées. Si personnaliser et identifier l'expérience humaine de la migration permet d'aller au delà des catégorisations sociales en vigueur dans l'espace public pour créer une "condition du migrant qui associe expérience et pensée de la frontière [permettant de] tirer un point de vue décentré sur le monde " (Agier, 2016, emplacement 638 sur 776) peut-on affirmer pour autant que ces écritures transforment leurs auteures?

4 Le corpus sur lequel s'appuie cet article est extrait de celui d'une recherche (Leroy 2017), composé à la fois de récits oraux - ethnographiques -, d'éléments scripturaux ordinaires produits par les femmes interrogées tels un agenda ou un cahier mais également de récits de vie (publiés ou non). Deux récits autobiographiques ont été 
extraits du fonds de l'A.P.A. ${ }^{1}$ (Association pour l'autobiographie et la conservation du Patrimoine Autobiographique) qui collecte et conserve des récits personnels.

5 Ma contribution prendra appui sur cette recherche traitant des rapports aux écritures de femmes migrantes hispanophones, qu'elles soient lettrées ou non. À travers des expériences singulières et hétérogènes, des histoires de vie dans le sens de Ferraroti (1983) et de également Pineau \& Le Grand (2013), je tenterai de rendre compte de la manière dont ces femmes disent s'être emparées, ou non, de l'écriture comme outil et moteur de transformation personnelle. Sur un autre plan, je verrai comment leurs nouveaux usages et pratiques des écritures les transforment et les "auteurisent", démentant parfois leurs propres déclarations.

\section{Un corpus hétérogène}

6 Comme dans tout classement visant à distinguer et séparer, des glissements ou des chevauchements sont intervenus entre les trois types d'autobiographies sélectionnées (orales, littéraires, écritures dites ordinaires) : un récit non publié le deviendra, une source littéraire sera complétée par un entretien oral, etc. Ces chevauchements indiquent la porosité relative de cette catégorisation et les mouvements qui s'opèrent, au fil du temps.

\section{Une collecte de récits oraux}

$7 \quad$ Les premières entrevues ont été réalisées principalement ${ }^{2}$ par l'intermédiaire des Cours Municipaux d'Adultes (C.M.A.) de la ville de Paris qui accueillent un nombre important d'auditeurs $^{3}$ de plus de dix-huit ans dans divers secteurs de formation (treize domaines répertoriés), dont des formations en français. En me présentant à quelques séances d'une école précise, j'ai pu contacter des femmes hispanophones apprenantes en français et désireuses de raconter leur histoire. Trois ont finalement poursuivi cette élaboration, une quatrième -qui disait refuser de s'inscrire à ces cours- a également accepté ces rencontres. Issues de différents pays d'Amérique latine (Colombie, Bolivie et Pérou), elles étaient âgées entre 32 et 57 ans au moment de notre première entrevue. Quelques points communs les rassemblent: elles ignoraient le français en arrivant en France, avaient une situation administrative précaire à leur arrivée et exerçaient différents emplois dans des domaines de faible qualification (aide à la personne, garde d'enfant, ménage). Elles ne vivaient pas en couple et avaient eu précédemment un ou deux enfants dans leur pays d'origine qui n'ont pas fait le voyage avec elles (une seule fera venir son fils par la suite). Elles souhaitaient toutes obtenir soit une régularisation de leur situation, soit accéder à la nationalité française et ont fait ou faisaient des démarches pour y parvenir.

8 Les différents entretiens se sont déroulés à leur domicile et/ou dans des espaces choisis par elles, sur une durée d'environ un à trois ans et ils perdurent pour certaines au-delà. Les discours me sont adressés directement par le prisme d'une relation ethnographique ${ }^{4}$ que l'on pourrait qualifier de chaleureuse voire d'amicale (LacosteDujardin, 1977) mais qui a débuté par une présentation du projet de recherche, dans un contexte de cours de français. Les enjeux de présentation, de langue sont donc inhérents à la situation provoquée et vont alimenter les formes de ces récits (entretiens enregistrés et retranscrits et observations participantes). 
9 Des éléments matériels scripturaux vont être collectés lors de nos rencontres : carnets, cahiers, feuilles, bibliothèques, etc. Ils serviront parfois d'appuis ou de démentis aux déclarations de pratiques. Ils alimenteront et complexifieront les discours produits.

Ce que l'on peut noter à la vue de ces différents documents est l'importance accordée à ces traces, même lorsqu'elles sont anciennes (l'une d'elles, Anna, conserve des cours de plus de vingt ans), elles sont rangées, classées à portée de main, consultables et consultées (étagères, bibliothèques). S'inscrire à un cours du soir est non seulement un investissement de temps (aller en cours après le temps de travail), mais représente un projet personnel majeur, visible dans leurs intérieurs.

11 L'un des premiers bouleversements évoqué est leur statut d' « auditrices ${ }^{5}$ » puisqu'elles possèdent une carte qui témoigne de cette activité (que certaines d'entre elles qualifient d'étudiante $\left.{ }^{6}\right)$. Accéder à cette inscription - les demandes sont nombreuses et les places comptées - représente un premier bouleversement dans leur rapport au savoir et plus particulièrement à l'écrit. Aller «à l'école » ou y retourner est déjà le témoignage d'une transformation en cours: revanche scolaire et/ou envie de promotion sociale, il s'agit d'un acte qui pose le changement envisagé dans leurs vies. Les traces des cours anciens et leur usage (l'une d'elle dit s'entrainer à refaire les exercices), bien après la suspension de leur activité en sont le témoignage.

\section{Collecte d'autobiographies abordant le rapport à l'écriture}

12 Une autre source de ce corpus provient des archives de l'A.P.A., où j'ai recherché des récits autobiographiques de femmes hispanophones, évoquant le rapport à l'écrit de leurs auteures.

13 Deux récits, là encore très distincts, seront extraits de ce fonds : celui d'une femme lettrée (doctorat en mathématiques) et celui d'une femme nouvellement lettrée (qui apprendra à écrire à l'âge adulte en France). Le premier sera publié ultérieurement en français par la volonté de son auteure et donnera naissance à une carrière d'écrivaine (romans, récits ${ }^{7}$ ).

14 Le fil du milieu, de Maria London (2003), s'apparente à une quête des origines familiales. Maria est chilienne, issue de deux familles de migrants de l'Europe de l'Est du début du $\mathrm{XX}^{\mathrm{e}}$ siècle. Elle retrace l'histoire de ses grands-parents et évoque simultanément le présent : sa propre situation de migrante en France pour suivre son mari au milieu des années 1970. L'écriture du récit de migration de ses ancêtres, réactive sa propre expérience et cherche un écho dans une mémoire difficile à reconstituer.

15 Le récit d'Isabel Gille, Andalouse en Bourgogne (2017), intervient alors qu'elle apprend le français après plusieurs décennies passées en France. Elle est Andalouse comme le suggère le titre et exilée de guerre : sa famille et elle-même ont fui la guerre civile tout d'abord en Espagne puis en France. Elle est hébergée avec sa mère et l'un de ses frères dans un camp de réfugiés en Bourgogne, qui sera dirigé par les Allemands pendant l'Occupation. Elle trouve du travail dans la région comme ouvrière agricole et y fonde une famille avec un Français. La rédaction de son récit qui la propulse dans l'écriture à un âge mature (ses enfants ont déjà quitté le domicile familial) lui prendra plusieurs années.

16 À travers ces deux récits, l'importance de l'acte d'écriture est indéniable pour chacune des auteures. Il est la trace de leur manière de se saisir d'une histoire de leur propre 
point de vue. L'appropriation des récits -qu'il soit familial ou individuel- reconfigure la place de chacune dans une Histoire. Elles prennent position et affirment leur manière de penser le monde, au risque de l'affrontement et du conflit avec leurs proches. Maria tente de dénouer les fils d'un secret familial dont on la somme depuis toujours de maintenir le silence. Réécrire cette histoire c'est indiquer sa distance et son opposition avec la norme en vigueur dans son environnement, la terreur de la parole, qui résonne politiquement de manière assez sourde. Lever le voile c'est se séparer d'un système narratif en conformité avec son milieu. Cette quête des origines, bouleverse son présent et réinterroge les relations qui tissent son existence.

Il y a une prise de risque manifeste - qui se traduira en conflit réel - et qui indique une transformation: elle s'émancipe d'un code familial pour construire un sens qui lui est propre. Elle s'auteurise filialement: elle ne s'insère pas docilement dans un récit préexistant, elle cherche (documents, témoignages), doute, construit et énonce les éléments d'une histoire qu'elle s'approprie et dans laquelle elle existe.

Pour Isabel l'écriture est une double découverte : à la fois un moyen de se raconter mais également de mettre en lumière la richesse de ses expériences. Son parcours d'exil et la description des multiples apprentissages qu'elle a dû cumuler, rompent avec l'idée qu'elle ne saurait que peu de choses puisqu'elle n'a pas été à l'école, comme son mari et ses enfants. Mais, et c'est ce qui me semble le plus important, elle ose, timidement en fin de récit évoquer son statut de femme isolée et reléguée aux tâches domestiques. Elle énonce clairement et presque sans aucun pathos la difficulté de ne jamais avoir pu décider seule du cours de sa vie, en qualité de fille, femme, étrangère, ouvrière agricole (quasiment domestique) puis même de femme mariée. L'écriture lui donne le moyen d'exprimer ce qui n'est pas vu ni entendu des autres : sa réflexivité. Elle s'empare d'une voix toute nouvelle: la sienne. Elle ose, s'auteurise à raconter la perception de son quotidien. Le risque vis-à-vis de ses proches est tout aussi grand que pour Maria London. Non seulement elle manifeste qui elle est mais signale simultanément que personne ne s'en est soucié auparavant. Il s'agit presque d'une émergence, d'un soi qui jaillirait là on ne l'attendait pas.

\section{Le genre comme unité signifiante}

Me centrer sur des écritures de femmes n'est pas en corrélation directe avec la toute contemporaine et très relative "découverte" d'une migration féminine ${ }^{8}$ mais vise plutôt à l'interroger et à pointer un phénomène ancien et structurel d'invisibilisation. Cette invisibilisation a certainement contribué à l'émergence de pratiques sociales distinctes, comme celles de l'écrit.

En effet, jusque-là délaissée ou reléguée dans l'ombre, la soudaine « découverte » d'une problématique féminine de la migration au début des années 2000 questionne. Même au plus faible de leur représentativité en France (en 1931) les femmes migrantes représentent malgré tout $40 \%$ des migrants et l'équilibre homme-femme avait été proche précédemment ( $47 \%$ en 1911). Le passage à une majorité - $51 \%$ en 2008 - n'est donc pas vraiment un grand écart avec les flux précédents et plus anciens ${ }^{9}$. Cependant, elles ont longtemps été pensées comme "à la marge ", voire absentes d'un phénomène de mobilité où l'homme agirait seul dans un premier temps avant d'être rejoint par le biais du regroupement familial ${ }^{10}$. Les femmes sont donc évoquées comme "agies " par le masculin qui déciderait et s'emparerait seul des trajectoires familiales. Quant à leur 
nombre (proche de la parité depuis fort longtemps), il est souvent occulté, comme s'il n'avait pas la même importance que celui de leurs homologues masculins ${ }^{11}$.

Même les situations d'apprentissage linguistique ${ }^{12}$ sont une manière de les laisser dans l'ombre. En effet, la forme contractuelle d'obligation linguistique et civique- injonction imposée aux migrant.e.s voulant s'installer durablement en France et n'ayant pas un niveau suffisant en français donne à voir une politique linguistique qui vise explicitement à l'autonomie des femmes, en présupposant de ce fait leurs dépendances. Camille Gourdeau, évoquant la double tension intégration/injonction, constate que " ces représentations occultent la diversité des trajectoires, des origines sociales et nationales ou encore des situations matrimoniales des migrantes " (Gourdeau, 2015, 26). Ce constat est certainement à rapprocher des assignations - au sens de stigmates de Goffman (Goffman, 1975) qui renforcent les catégorisations.

Les femmes rencontrées ou évoquées dans cette recherche ont en écrasante majorité migré seules et ne correspondent que très peu aux stéréotypes énoncés ci-dessus. Si elles subissent des dominations en lien avec la figure masculine, toutes leurs histoires démontrent leur volonté et les moyens qu'elles se donnent pour s'en émanciper (séparations, migration, emplois, etc.) dont l'accès à l'écriture et au récit font partie, mais de manière volontaire et solitaire.

En sus des procédés de stigmatisation liés à la migration, cette manière de se représenter uniformément un groupe construit sur la base d'un sexe social occulte donc toute singularité des personnes et comporte le danger de l'essentialisation d'un groupe. Les formes d'apprentissages et d'appropriations linguistiques et scripturales sont au cœur de ses constructions stéréotypées. Les autres composantes des provenances et configurations sociales (culturelles, économiques, géographiques) mais également des trajectoires personnelles en terme d'apprentissage et d'expérience (formelle, non formelle, familiale, intime, etc.) sont ainsi oubliées. C'est pourquoi on peut postuler que la restitution de la particularité des parcours individuels et de leur dimension complexe permet de penser au-delà des assignations globalisantes.

\section{Récits de l'intime}

Une donnée constante, et réitérée à chacune de mes recherches depuis 2005, montre que les femmes ont un discours sur leurs rapports à l'écrit qui les lient à l'intime. Même lorsqu'elles sont salariées et qu'elles mobilisent l'écrit dans leur activité, une grande proportion de récits se focalise sur la vie familiale en relevant les incidences de places et statuts au sein des couples que l'accès à certains usages bouleverse (chéquier, gestion administrative du foyer). Ainsi, accéder au chéquier et à la gestion des dépenses de la famille permet d'obtenir un droit de regard et de choix sur ce qui est possible, légitime ou non d'acheter. La connaissance du montant des charges et ressources, leur nature (allocations) procure parfois une indépendance financière, jusque là impossible. De même, la signature de certains documents administratifs libère d'une dépendance visà-vis de l'autre partenaire, libération qui est potentiellement source de conflits puisque redéfinissant par là-même les places et rôles de chacun.e au sein de la famille. Les femmes décrivent ainsi les problématiques de pouvoir des écritures domestiques. Ces problématiques avaient déjà été dépeintes dans le contexte soninké par Muriel Azoulay et Catherine Quiminal (2002) avec l'émergence de ces « femmes réveillées » qui soudain bousculaient l'ordre établi par de nouvelles compétences et la volonté d'en user. La 
transformation par l'écriture passe aussi par ces écrits courants, administratifs mais qui permettent de prendre du pouvoir sur le quotidien et d'exercer à ce titre son librearbitre.

D'autres récits témoignent du lien ténu que ces femmes entretiennent, depuis leur migration (elles disent ne pas avoir eu de pratiques semblables auparavant) avec des écritures à la première personne, même si celles-ci relèvent d'écritures dites ordinaires (cahiers, carnets, etc.). Elles y relatent des faits plus ou moins habituels de leur vie de tous les jours (achats, sorties, adresses, recettes, visites, etc.) et conservent ces récits hors de portée d'autrui. Pour l'une d'entre elle - Monica - c'est même un rituel extrêmement ordonné (jours et heures précises d'écritures et de relectures) qui s'apparente à ce que Foucault (2008) désigne comme une technique de soi. Ces écritures font trace de leur histoire et elles peuvent y avoir recours pour vérifier ou se remémorer un moment important.

C'est une manière de s'approcher du "gouvernement de soi » de la maîtrise de soi que Foucault préconisait à travers ces techniques. Il semble alors que ce pouvoir sur soi même pourrait s'apparenter à l'idée d'une auteurisation, une manière de prendre du pouvoir sur soi par l'acte d'écriture.

\section{Violences sexuées}

Les rapports de domination masculine au sein de la cellule familiale sont le point commun des récits collectés, parfois même ils sont exercés avec l'aide de certaines femmes de l'entourage (mères, belle-mère, belle-sœur). Les violences exercées à l'encontre de ces femmes, en raison de leur genre traversent tous les milieux et si leurs formes et leurs évocations varient, elles sont comparables en terme de dépréciation et d'iniquité. Elles sont en outre accentuées par l'accord tacite de hiérarchisation sexuelle des sociétés patriarcales dans lesquelles ces femmes évoluent. La violence individuelle ordinaire se trouve renforcée par l'impunité, voire le soutien collectif qui l'accompagne le plus souvent. Anna ${ }^{13}$ sera par exemple mise à la porte de la maison familiale à l'annonce de sa grossesse dont le petit ami renie toute implication; contrainte à de multiples travaux domestiques comme bonne à tout faire, dans des familles où de nouveau la domination sexuelle la renverra, avec sa fille à la rue (renvoyée sur le champ par une patronne quand Anna dénonce auprès d'elle une tentative de viol du mari). La migration en France ne résoudra pas la situation : elle vivra dans des rapports d'oppression constante avec le père de son deuxième enfant né en France et dont plus de vingt ans après elle pense être la seule responsable de la naissance («je ne fais attention »). Isolée à son arrivée (elle ne parle pas un mot de français), elle accepte de sortir avec un voisin qui parle un peu espagnol :

\footnotetext{
Je trouve toujours le papa de mon fils. Et un jour je suis partie avec el. J'ai couché avec el y voilà. $Y$ je ne fais attention y je fais à mon fils. Après ma sour est très fâchée, fâchée, fâchée, je souffris beaucoup. Parce que je suis allée à l'hôpital y je ne sais rien du tout. Je ne parle pas (...).
}

Ces phénomènes de violences sont renforcés, voire alimentés par le statut socioéconomique et du statut administratif de la migrante (dite « avec » ou " sans papiers ", arrivée en France légalement ou non, etc.). La situation de dépendance est alors propice à des abus de pouvoir de tous ordres (sexuels, monétaires, physiques, familiaux, etc.). Bien évidemment, une concurrence de violences vient s'abattre sur celles qui sont les 
plus désargentées, les plus dépendantes statutairement et les plus isolées linguistiquement. «Je ne sais rien du tout, je ne parle pas » dit Anna lorsqu'elle évoque sa première visite à l'hôpital. Elle semble comme paralysée à la fois par une situation qu'elle subit (nouvelle grossesse non désirée) et par l'incapacité à l'exprimer à d'autres. On pourrait alors penser à une double absence de pouvoir sur soi.

De manière plus précise, les femmes interrogées sur leurs rapports à l'écrit ont systématiquement et longuement dépeint des situations dont elles étaient les victimes en raison surtout de leur sexe social. Ces témoignages, qui pouvaient de prime abord paraître en dehors du sujet, et leur manière constante d'y revenir agissent bien comme une forme de bannière identificatoire, où, derrière le récit, s'entend la volonté de se dire au monde et d'exposer ses souffrances.

\section{Discriminations, déqualifications et « auteurisations »}

30 La parole dans l'espace public de personnes peu ou pas valorisées socialement est difficilement accessible et répandue. Ainsi la vie quotidienne d'Anna, les problèmes auxquels elle est confrontée sont rarement évoqués et médiatisés par ceux et celles qui les éprouvent. Judith Butler, lors d'une conférence donnée à l'Université Paris 8 à l'automne 2015, l'évoque à travers la notion de reconnaissance (partielle ou non). Cette dernière serait à même de tempérer la violence faite aux personnes invisibilisées par des rapports de pouvoir en leur défaveur. Le récit devient le moyen d'exposer la déqualification en usage et sert d'appui à une transformation. C'est l'exposition narrative qui devient le pivot du changement. Changement simultané à la fois des personnes concernées - elles ne s'appréhendent plus seulement comme victimes ou objets sociaux mais bien comme héroïnes des récits et sujets de leur vie - et changement dans les représentations des tiers qui perçoivent des personnes avec des histoires et non plus des catégories d'individus sans âme. Cette bascule représentative serait à même de transformer radicalement les rapports sociaux.

31 Ce travail de reconnaissance par le récit s'apparente à la longue tradition philosophique sur le lien entre absence de reconnaissance (déni ou mépris) et émergence de conflits sociaux. C'est en terme de luttes sociales que se positionne la troisième génération de l'école de Francfort qui postule le besoin d'une triple reconnaissance, à la fois pour prévenir les violences mais également afin de bouleverser les rapports sociaux en offrant un cadre critique. S'appuyant sur Hegel et Mead, Honneth $(2000,208)$ évoque l'amour, le droit et la solidarité (ou l'estime sociale) comme les trois formes permettant la confiance en soi, le respect de soi et enfin l'estime de soi ${ }^{14}$, comme le triptyque et socle de cette métamorphose annoncée.

Cette mutation est lisible dans le texte d'Isabel Gille. Elle prend la décision de produire son récit à partir du moment où son expérience est considérée comme ayant de la valeur par un tiers qui l'entend : «ça vaudra la peine d'être lu », lui dit une personne à l'accueil d'un centre social à qui elle raconte son parcours. Traduire son expérience par le récit permet d'y porter un intérêt et donc de se sentir digne d'intérêt. Une reconnaissance sociale à la fois comme valorisation personnelle de son parcours individuel, mais également d'un groupe d'appartenance qui soudain sort de son invisibilité : " Nous sommes les "sans profession" ", écrira-t-elle en évoquant le travail féminin domestique (Gille, 2017, 309). 


\section{Temporalités et visée transgénérationnelle des récits}

33 Si tout discours est situé, il comporte en revanche une portée historique indéniable. Il est produit pour s'approprier ou se réapproprier un cheminement et vise à le transmettre à autrui. Il parle dans le temps présent d'un passé qui veut témoigner au futur. Les temps sont toujours entremêlés dans les récits évoqués, leur volonté de transmission les rend comme des passeurs temporels.

\section{Maria London : des ancêtres aux petits-enfants}

Son récit Tisseuse de mémoires de la Patagonie aux Balkans est la reconstitution de l'histoire familiale - maternelle et paternelle - venue de l'Est (Ukraine, Crimée et Croatie) au début du XXe siècle. Elle y retrace le parcours migratoire de deux familles qui se rejoindront pour composer les deux branches de la sienne - en menant une véritable enquête sur les différents protagonistes. Cette enquête, avec recherche d'informateurs et d'informations, s'avère une véritable quête de sens. Pour quelle raison migrer? Poser cette question vis-à-vis de ses aïeux est une forme de réactualisation de sa propre problématique de migrante. La quête du passé reformule donc au quotidien sa propre expérience. Le prologue pose ainsi d'emblée la question du manque et de la perte :

"Je dédie ces pages à tous ceux qui, descendants d'immigrants du monde entier, ont ressenti l'envie de regarder en arrière, de s'interroger sur leurs racines restées sur un autre continent, et qui ont découvert avec tristesse, qu'une partie de ces racines était pour toujours hors de terre » (London, 2003, 9).

À la fin de l'ouvrage, Maria s'aperçoit de l'écart entre sa visée initiale et le résultat produit en elle et chez ses proches :

«Je croyais qu'en racontant mon histoire j'allais guérir ma nostalgie et je découvre qu'elle n'a fait que grandir. J'ai écrit guidée par le désir de partager avec mes enfants la part de vie qui leur revenait à travers moi et, eux, ils n'ont rien voulu lire » (London, 2003, 227).

La publication d'El hilo del medio a de plus été douloureuse pour l'auteure vis-à-vis de ses ascendants. Maria London évoque le retour très critique et acerbe de sa famille :

«Le livre disparut des rayons des librairies de la ville, non qu'il fut vendu mais parce qu'il fut censuré !!! Ma famille maternelle ne me pardonnait pas d'avoir abordé certains sujets (intimes il est vrai mais remontant à un demi-siècle ou plus). » (London, 2014, 89).

Alors qu'elle-même n'a pas l'impression de révéler des secrets, les tantes ont lu derrière les lignes de Maria London un secret que l'auteure ignorait à l'époque de la sortie de l'ouvrage (la liaison amoureuse cachée d'une aïeule) et dont la pseudo révélation (les tantes lectrices ont cru -à tort- que Maria London y faisait allusion) provoque le courroux général, rendant l'ouvrage inaccessible au Chili. Quant aux enfants auxquels était dédié cet ouvrage, sa langue, l'espagnol, aura été un empêchement à la lecture et il faudra attendre sa parution en français pour qu'elle soit possible.

\section{Isabel, dédicace et transformation?}

L'expérience d'Isabel Gille est très différente. Tout d'abord, elle rédige en secret, puis elle se ravise : 
«J'avais pensé que ce cahier resterait dans un tiroir, que mes enfants le découvriraient après ma mort. Finalement je ne suis pas mécontente que les choses se soient passées ainsi. J'ai pu connaître leurs réactions ainsi que celles des autres personnes qui ont eu l'occasion de lire mon histoire ». (Gille, 2017, 317).

Des similitudes importantes demeurent dans la volonté de restituer des scènes quotidiennes d'un passé révolu et méconnu des jeunes générations ou des habitants du pays de résidence. Le récit est une manière de valoriser à la fois l'expérience personnelle, mais également les difficultés suscitées par la migration. Isabel en décrivant les scènes ordinaires auxquelles elle a été confrontée, tente de mettre en avant un point de vue, pourtant très distancié, dont elle suppose qu'il n'a pas été pris en considération. C'est une forme de réhabilitation de la vie ordinaire de personnes souvent minorées et de la place des femmes. Ainsi les scènes de la vie agricole, des coutumes religieuses ou du travail féminin domestique et des rapports genrés au sein de la famille se retrouvent-elles dans chaque récit et marquent le passage d'un monde à un autre, d'une société à une autre. Elles marquent le temps et l'histoire du protagoniste dans des changements ou bouleversements importants. Dans la volonté d'« écrire sa vie », il y a la volonté d'en construire le sens pour soi mais aussi pour autrui.

\section{Conclusion}

Les histoires de vie permettent d'œuvrer contre les catégorisations et les stéréotypes en pointant, à travers l'ordinaire du récit autobiographique, des expériences remarquables et exceptionnelles de personnes distinctes. Paradoxalement, elles permettent d'effectuer des rapprochements expérientiels en montrant comment des pratiques, qui paraissent à première vue singulières, sont partagées par un groupe qui ne se reconnaissait pas comme collectif. Il en est ainsi de certaines pratiques d'écritures qui se réalisent et s' « auteurisent » au-delà du clivage habituel de la théorie du grand partage entre lettrées et non lettrées. Ainsi, chacune à leur manière, Isabel Gille, la tardive apprenante en alphabétisation et Maria London, la docteure en mathématiques, rompent avec le schéma de silence qui leur a été imposé depuis l'enfance en produisant des récits auteurisant leur propre parole. Cette transformation personnelle provoque des changements, voire des bouleversements avec leurs proches.

41 D'autres transformations moins visibles car produites dans l'ombre et le secret sont pourtant à même de modifier le rapport au monde de leurs auteures. Souci de soi ou récit testimonial comme héritage pour le futur, reprendre le sens de son histoire et la propriété de son récit, devient une manière quotidienne d'entrevoir la pratique scripturale. Que les récits soient exposés ou cachés, leur réalisation provoque pour leur auteure une appropriation du discours sur soi à même de produire une subjectivation, sorte d'herméneutique de soi, proche de ce que Foucault (2008) décrivait. Parfois aussi, l'inscription à un cours, la pratique d'une formation linguistique choisie peut, par le basculement de représentations qu'elles occasionnent (devenir étudiante, aller à l'école), être à même de valoriser la personne à ses propres yeux et de la renforcer dans sa capacité d'agir. Se remémorer et s'appuyer sur ces moments (traces de cahiers ou récits) sont des moyens de conforter et confirmer auprès de soi même comme d'autrui, le mouvement entrepris. 

équivalentes non plus. Les facilités économiques, l'appartenance socio-culturelle, la composition familiale, l'âge, le niveau d'étude, l'écart de ces données avec les dominateurs et la manière singulière d'y répondre créent des circonstances et des positions différentes face à la société. Cependant ce n'est pas un cloisonnement des situations qui fait sens, mais plutôt, à travers cette complexité d'appartenances, des zones inattendues de rapprochement notamment par des modalités d'exclusion qui se réitèrent dans le temps. Je pense ainsi, par exemple, à la prime au retour, dont Isabel Gille dépeint une forme plus ancienne mais assez similaire dans les années 1940. Il existe dans tous ces récits un désir très fort de reconnaissance par le témoignage pour autrui, permettant de faire évoluer sa propre expérience, non plus uniquement en qualité de victime, sujet balloté par l'histoire, mais bien comme auteure de son parcours.

Si à l'instar de Paul Ricoeur $(1994,15)$ « on peut adopter comme hypothèse de travail que la souffrance consiste dans la diminution de la puissance d'agir », la mise en récit de son expérience réattribue alors à son auteure un pouvoir d'agir (agency), promesse et socle de transformations choisies.

\section{BIBLIOGRAPHIE}

Agier, Michel (2016) Les migrants et nous. Comprendre Babel, Paris, CNRS éditions, 776 emplacements (version numérique).

Azoulay, Muriel ; Quiminal, Catherine (2002) Reconstruction des rapports de genre en situation migratoire. Femmes "réveillées", hommes menacés en milieu soninké, VEI Enjeux, n 128, pp. 87-120 (Rapports de sexe, rapports de genre, domination et émancipation).

Beauchemin, Cris ; Borrel, Catherine ; Regnard, Corinne (2013) Les immigrés en France : en majorité des femmes, Populations et Sociétés, n 502 [réf. du 12 nov 2016] [Disponible sur Internet]

Butler, Judith (2015) Le genre en traduction autour de (et avec) Judith Butler, Université Paris VIII, Conférence du jeudi 12 novembre 2015.

Dallera, Corinne ; Ducret, Véronique (2004) Migration Féminine, au-delà des stéréotypes, Genève, Le 2ème Observatoire, 108 p. [Disponible sur Internet

e-Migrinter, 16 | 2017 
Damgé, Mathilde (2016) Idée reçue sur les-migrants 6-6 : "le regroupement familial est la porte d'entrée d'une immigration-massive", Le Monde (12/10) [réf. du 27/11/2017] [Disponible sur Internet]

Delory-Momberger, Christine (2014) De la recherche biographique en éducation. Fondements, méthodes, pratiques, Paris, Téraèdre, $274 \mathrm{p}$.

Fassin, Éric (2015) D'un langage l'autre. L'intersectionnalité comme traduction, Raisons politiques, $\mathrm{n}^{\circ} 58$, pp 9-24.

Ferrarotti, Franco (2013) Histoires et histoires de vie, Paris, Téraèdre, 195 p.

Foucault, Michel (2008) Le gouvernement de soi et des autres. Cours au Collège de France, 1982-1983, Paris, Gallimard/Le Seuil, 400 p.

Gille, Maria Isabel (2017) Andalouse en Bourgogne, in Leroy, D. (dir.) «- Des romans ? - Non : leurs vies! » Écritures et Auteurisations de femmes migrantes hispanophones, Saint-Denis, éditions Connaissances et Savoirs, pp. 187-319 (Actes graphiques).

Goffman, Erving ; Kihm, Alain [trad.] (1975) Stigmate. Les usages sociaux des handicaps, Paris, Les éditions de Minuit, $170 \mathrm{p}$.

Gourdeau, Camille (2015) Une politique d'intégration au service des femmes étrangères? L'exemple français du CAI, Hommes et Migrations, $n^{\circ} 1311$, pp. 23-29. (Femmes et migrations).

Honneth, Axel ; Rusch, Pierre [trad.] (2000) La lutte pour la reconnaissance, Paris, Éditions du Cerf, $232 \mathrm{p}$.

Lacoste-Dujardin, Camille (1977) Dialogue de femmes en ethnologie, Paris, Maspéro, $114 \mathrm{p}$.

Le Courant, Stefan (2014) “Le jour où on a découvert l'Europe". Le voyage et l'aventure dans le récit d'Ibrahim Kanouté, Hommes et migrations, n 1306, pp. 73-80 (Écrire la migration).

Leroy, Delphine (2014) Saisir, transcrire, traduire des récits de vie de migrantes hispanophones, Hommes et migrations, $\mathrm{n}^{\circ}$ 1306, pp. 93-100 (Écrire la migration).

Leroy, Delphine (2017) «-Des romans? - Non : leurs vies!» Ecritures et Auteurisations de femmes migrantes hispanophones, Saint-Denis, éditions Connaissances et Savoirs, 462 p. (Actes graphiques).

Leroy, Marie ; Weirich, Anna (2014) Chercheures ... en migration ? Échanges d'expériences, Hommes et migrations, $n^{\circ} 1306$, pp. 83-90 (Écrire la migration).

London, Maria (2003) Tisseuse de mémoires de la Patagonie aux Balkans [El hilo del medio, Paris, L'Harmattan, $230 \mathrm{p}$.

London, Maria (2014) Témoignage, in Leroy, D. ; Leroy, M. (dir.) Histoires d'écrits, histoires d'exils. Perspectives croisées sur les écritures en migration(s), Tübingen (Allemagne), Editions Narr, pp. 97-90.

Morokvasic, Mirjana (2008) Femmes et genre dans l'étude des migrations : un regard rétrospectif, Les Cahiers du CEDREF, $\mathrm{n}^{\circ} 16$, pp. 36-58 (Femmes, genre, migrations et mondialisation).

Pineau, Gaston ; Le Grand, Jean-Louis (2013) Les Histoires de vie, Paris, PuF, 128 p. (Que sais-je ?), 5ème édition.

Ricœur, Paul (1985) Temps et récit 3, Paris, Le Seuil, 533 p.

Ricœur, Paul (2013) La souffrance n'est pas la douleur, in Marin, C. ; Zaccai-Reyners, N. ; et al., Souffrance et douleur. Autour de Paul Ricoeur, Paris, PuF, pp. 13-34. 


\section{NOTES}

1. L’A.P.A. a été créée par Philippe Lejeune en 1992. Son but est de permettre aux textes autobiographiques quels qu'ils soient (carnets, récits non publiés) d'être accueillis, conservés, lus, recensés, étudiés. Les textes déposés à l'association sont tous lus et chacun d'entre eux est l'objet d'un compte rendu publié ensuite dans le catalogue annuel, appelé le "Garde-mémoire", consultable en ligne et à la Bibliothèque nationale de France (B.N.F).

2. Une seule femme n'a pas été approchée par cet organisme. Mais elle intervient comme unité signifiante de ce type de formation, à laquelle elle dit refuser de s'inscrire. Paradoxalement, très peu d'éléments sont visibles, étudiés et consultés dans ces structures sur les personnes qui ne participent à aucun dispositif institutionnel de formation linguistique.

3. En 2011, Les Cours Municipaux d'Adultes (C.M.A.), institution historique de cours pour adultes dans la capitale, ont accueilli dans l'année près de 30000 auditeurs, tous secteurs confondus (secteur des langues, enseignement général, technique, entreprises, artistique etc.). Ces personnes se sont réparties dans 140 établissements scolaires, avec une équipe de 850 enseignants dont 122 professeurs dans le seul secteur alphabétisation. Pour les formations en français, 8000 apprenants (sur les 15000 personnes formées en français par la ville de Paris) le sont par le biais des C.M.A. La plupart des cours ont lieu dans les locaux d'écoles élémentaires, après le temps scolaire.

4. Camille Lacoste-Dujardin exprime l'ambivalence de la relation avec Me Laali, son « enquêtée » qui ressemble pour beaucoup à celles entretenues avec les différentes femmes rencontrées : «Ce type de rapport particulier qui lie l'enquêteur à l'enquêté dans le domaine de l'ethnologie pose, en fait, bien des problèmes. Il paraît bien souvent très déséquilibré entre les deux parties en présence qui y investissent des contenus très différents. Sans doute Me Laali et moi n'échappons nous pas à ce déséquilibre. Il semble cependant que nous ayons établi une relation réellement amicale et durable (depuis maintenant six ans) dans le respect et l'estime mutuels. Nous nous sommes je crois aussi beaucoup influencées l'une l'autre. » (Lacoste-Dujardin, 1977 : 114)

5. Nom donné par la Mairie de Paris aux personnes inscrites aux Cours Municipaux d'Adultes.

6. Cet élément est souvent produit comme élément de "preuve » d'intégration et de niveau de langue dans les dossiers de demandes de régularisation et/ou d'accès à la nationalité française.

7. Il n'est pas fait mention explicite aux autres ouvrages de Maria London dans cet article car ils évoquent beaucoup moins sa relation à l'écriture.

8. Nombre d'études ou d'articles pointent à la fois l'absence d'intérêt ancien pour ce thème, et l'intégration partielle de cette donnée dans les nouveaux textes, qui soulèvent la "nouveauté " d'une situation existant depuis longtemps mais fort peu étudiée et médiatisée jusqu'alors. La «nouveauté » est donc du côté de l'intérêt, pas de la situation : «La migration féminine n'est ni récente, ni marginale, pourtant les conditions de vie des femmes et les rapports sociaux de sexe n'ont été pris en compte que tardivement par la littérature sur les migrations. » Dallera, Corinne \& Ducret, Véronique $(2004$, p. 3). De plus, dès l'introduction il est souvent demandé aux chercheures d'expliciter ce choix de thématique « On n'en finit pas de (re)découvrir les femmes en migration. Comme s'il fallait à chaque fois et toujours légitimer de s'être risqué-e dans ce champ de recherche resté longtemps en marge à la fois des études sur les femmes, le genre et les rapports sociaux de sexe, et des recherches sur les migrations. » Mirjana Morokvasic (2008, p. 36). 9. Cf. Beauchemin, Borrel \& Régnard (2013).

10. Aujourd'hui cette mesure ne concerne l'arrivée sur le sol français que d'environ 12000 personnes par an. En 2015 ce sont 11514 personnes qui en avaient bénéficié, contre 12121 en 2014. Sources : Ined, repris par Damgé dans Le Monde (2016).

11. On pourrait postuler que l'homme migrant est, en terme de représentation collective, plus anxiogène qu'une population mixte et voisine des résidents plus anciens. Pour preuve, cet engouement soudain pour l'épreuve des réfugiés autour de la médiatisation de la mort du petit 
Aylan Kurdi, cet enfant kurde retrouvé sur une plage turque en septembre 2015. La photographie d'un petit enfant décédé des suites d'un des nombreux naufrages de migrants en Méditerranée a soudain révélé au grand public que les réfugiés n'étaient pas uniquement des hommes, mais une population semblable à la nôtre, multiple et composite, digne de compassion, d'intérêt, d'empathie.

12. Le contrat d'intégration républicaine (CIR) est signé par tous les étrangers primo-arrivants admis pour la première fois à séjourner en France et qui souhaitent s'y installer durablement. Extrait du CIR: "C'est au cours de cet entretien que sont prescrites: la formation civique obligatoire, la formation linguistique. » La formation linguistique : «L'apprentissage du français est une condition impérative pour pouvoir s'intégrer dans la société française. (...) Une fois prescrite, cette formation est obligatoire. En la suivant avec assiduité et sérieux, et en progressant entre le test initial, le test intermédiaire et le test final, l'étranger respecte les conditions requises pour l'obtention d'une carte de séjour pluriannuelle, prévue par la loi du 7 mars 2016. » Des sanctions existent et s'appliquent en cas de non-respect des obligations du CIR (http://www.immigration.interieur.gouv.fr/Accueil-et-accompagnement/Le-parcourspersonnalise-d-integration-europeenne).

13. Anna (nom d'emprunt) est Colombienne et a 57 ans lorsque je la rencontre en 2013. Elle vit en proche banlieue avec son fils cadet né en France. Sa fille est demeurée en Colombie. Issue d'une famille d'agriculteurs pauvres elle n'a été que deux années à l'école. Elle a migré pour raisons économiques, laissant sa fille aux soins de l'une de ses sœurs. Elle obtient des papiers en juillet 2010 et interrompt ses cours d'alphabétisation car ils ne correspondent pas à ce qu'elle en attend (plus d'écriture), mais souhaite poursuivre son apprentissage dans l'informel (avec son neveu).

14. Honneth reprend Hegel dans les modèles de reconnaissance visant dans les interactions à ce que les sujets humains développent de nouvelles formes de relation positive à soi. « Les individus ne se constituent en tant que personne que lorsqu'ils apprennent à s'envisager eux-mêmes à partir d'un point de vue d'un "autrui" approbateur ou encourageant, comme des êtres dotés de qualités et de capacités positives. L'étendue de ces qualités et donc le degré de cette relation positive à soi-même, s'accroît avec chaque nouvelle forme de reconnaissance que l'individu peut s'appliquer à lui-même en tant que sujet. L'expérience de l'amour donne ainsi accès à la confiance en soi, l'expérience de la reconnaissance juridique au respect de soi et l'expérience de la solidarité, enfin, à l'estime de soi. » (Honneth, 2000, 208).

\section{RÉSUMÉS}

Comment et pourquoi des femmes migrantes se saisissent-elles de l'écriture en langue française aujourd'hui ? C'est à travers des écrits très dissemblables et des récits sur ces pratiques que sera envisagé une forme de puissance d'agir que pourrait susciter certains usages de l'écriture. Cette « auteurisation » transversale à des écritures ordinaires et littéraires (agenda hors d'âge, carnets, récits autobiographiques aux statuts divers, cahiers d'apprentissage du français) serait un moyen de dominer le récit de soi et d'être ainsi à même de le transformer à ses propres yeux comme à celui des autres. 
INDEX

Index géographique : France

Mots-clés : femmes, récit, histoire de vie, relations avec le pays d'accueil

\section{AUTEUR}

\section{DELPHINE LEROY}

MCF, Université Paris 8, EXPERICE - EA3971

Delphine.leroy05@univ-paris8.fr 